\title{
No nos dejes caer en tentación: Prototipo negativo de la mujer en las leyendas de Tonalá.
}

\section{Do not let us fall into temptation: Negative prototype of women in the legends of Tonala.}

Esta obra está bajo una Licencia Creative Commons Atribución 4.0 Internacional. DOI: $10.32870 /$ sincronia.axxii.n74.14b18

\author{
Alfredo Hermosillo López \\ Departamento de Humanidades y Artes / Universidad de Guadalajara \\ (MÉXICO) \\ mefiscatulo@hotmail.com \\ Jessica Marcelli Sánchez \\ Departamento de Humanidades y Artes / Universidad de Guadalajara \\ (MÉXICO) \\ cd.historia@cutonala.udg.mx \\ jessica.marcelli@cutonala.udg.mx

\section{Iván Pelayo Sánchez} \\ Departamento de Humanidades y Artes / Universidad de Guadalajara \\ (MÉXICO) \\ correo_electrónico@hotmail.com
}

Recibido: 02/10/2017

Revisado: $22 / 01 / 2018$

Aprobado: $17 / 02 / 2018$

\section{RESUMEN}

Este artículo analiza la leyenda "La bella de Puente Grande" como sustrato de memoria colectiva que da cuenta de experiencias compartidas generacionalmente y es usado para regir las creencias y el comportamiento de un pueblo. Para llevar a cabo este análisis se recurre al modelo actancial de Greimas y a la reconstrucción del imaginario simbólico presente en el relato.

Palabras clave: Leyendas. Memoria colectiva. Análisis literario-simbólico. 


\begin{abstract}
This article analyzes the legend "La bella de Puente Grande" (the Beauty of puente Grande) as a substrate of collective memory giving account of shared experiences generationally and used to rule the beliefs and behavior from local people. In order to carry out this analysis we use Greimas' actancial model and the reconstruction of the symbolic imaginary present in the story.
\end{abstract}

Keywords: Legends. Collective memory. Literary-symbolic analysis.

\title{
Introducción
}

Este artículo forma parte de un amplio proyecto que tuvo como objetivo el estudio del patrimonio inmaterial de Tonalá. Para llevarlo a cabo, tuvimos que buscar información que no se encontraba disponible a primera mano, así que empezamos realizando un trabajo compilatorio. Con este propósito recurrimos a la investigación documental y al trabajo de campo. La investigación documental se apoyó en documentos formales, tales como los obtenidos a través de fuentes bibliográficas, hemerográficas y, sobre todo, de la investigación archivística. En una segunda etapa realizamos trabajo de campo, que consistió en salidas a las diferentes localidades del municipio, con equipo para registrar y documentar entrevistas a miembros de comunidades específicas que trasmitieron su conocimiento sobre historias orales, mitos y leyendas de su comunidad, así como tradiciones artísticas de diversos géneros.

En lo que respecta a la catalogación y el estudio del patrimonio literario, se realizó el trabajo de compilación de un corpus de historias que reflejan el conjunto de creencias y convenciones culturales compartidas por la comunidad tonalteca. Una vez obtenido este corpus pudo procederse al análisis crítico de las formas literarias como representaciones de la actividad antropológicoimaginaria, pues éstas reflejan las visiones del mundo y los referentes sociales de una época dada. El análisis que presentamos es una muestra de ello, ya que está dedicado a explorar las manifestaciones del pensamiento simbólico mítico en los relatos populares; en este caso, las leyendas. 
El artículo está dividido en cinco apartados. En el primero explicamos brevemente el concepto de patrimonio cultural que tomamos como punto de partida, y la consideración de las leyendas como sustrato de memoria colectiva; en el segundo, el origen de la leyenda La bella de Puente Grande y su categorización como texto de advertencia moral. El tercer apartado presenta el análisis actancial, basado en el modelo de Julien Greimas, como paso previo al análisis simbólico mítico que realizamos en el cuarto apartado. Hemos elegido el modelo de Greimas porque éste permite mostrar el funcionamiento de cualquier relato y llegar a conclusiones verificables, lo más alejadas posible de la mera subjetividad. Finalmente, presentamos nuestras conclusiones en el quinto apartado.

\section{Leyendas y patrimonio cultural}

El patrimonio cultural inmaterial, en su sentido más amplio, está formado por bienes intangibles que la historia ha legado a una nación y que en el presente tienen una especial importancia histórica, científica, simbólica o estética. Es la herencia recibida por los antepasados que testifica de su existencia, de su visión del mundo, de sus formas de vida y de su manera de ser; está relacionada estrechamente con la memoria, la evocación al pasado y las formas tradicionales de vida.

Las leyendas transmiten un acervo de experiencias compartidas generacionalmente; pertenecen al corpus de textualidades de origen oral, colectivo y popular, creado y usado para regir las creencias y el comportamiento de un pueblo, para arraigar sus costumbres y tradiciones:

Este tipo de textualidad, por el hecho de estar funcionando en el seno de una cultura, se transmite mediante los mismos mecanismos por los que un pueblo transmite a las generaciones más jóvenes su herencia cultural: de boca en boca, a través del ejemplo en la práctica cotidiana, y también mediante textos escritos. (Pérez, 2001, p.56)

Este sustrato de memoria colectiva surge de la necesidad que tiene todo grupo humano de darse una imagen de sí mismo. Así pues, en las leyendas se revelan las figuras recurrentes por las cuales una cultura entera se reconoce. Su estudio nos permite acercarnos "a los espacios simbólicos en 
cuyo seno los individuos y las comunidades leen y construyen los ejes de su realidad". (García, 2007, p. 20)

\section{La leyenda de "La bella de Puente Grande"}

En un trabajo anterior (Marcelli, Pelayo \& Hermosillo, 2017) compilamos el corpus de leyendas que consideramos como patrimonio literario de Tonalá. Algunas de estas narraciones ya habían sido publicadas en el libro Leyendas de Tonalá, de Aurelio Nuño, que fue nuestro punto de partida para seleccionar los textos que cumplían con las características del género, luego les otorgamos una nueva forma literaria, más acorde con el género al que pertenecen, y pulimos su redacción para que puedan leerse con agrado.

Además de esta tarea de selección y reescritura de textos ya publicados, compilamos historias recogidas en entrevistas orales para darles una forma escrita. ${ }^{1}$ De este trabajo surgió $L a$ bella de Puente Grande, que es una leyenda de advertencia moral, es decir, un texto que señala cómo se debe vivir y comportarse en la vida cotidiana, que regula relaciones y enseña a someterse a las mismas normas, jerarquías, procedimientos y reglas.

\section{Análisis actancial}

Para Greimas, existen seis actantes o constantes funcionales en los relatos. Hay, en primer lugar, un objeto (de una petición, un deseo o una búsqueda) que hace pareja con un sujeto que pide, busca o desea este objeto; la siguente pareja está constiuida por el destinador que envía el objeto y el destinatario que lo recibe; finalmente están el adyuvante, que ayuda al sujeto en su búsqueda, y el oponente que se resiste a ello. Las relaciones entre objeto-sujeto, destinador-destinatario, y

\footnotetext{
${ }^{1}$ Las entrevistas orales también sirvieron para contrastar las leyendas publicadas con su versión oral y, en algunos casos, modificar la versión escrita.
} 
oponente-adyuvante forman el "modelo actancial". El Diccionario de términos literarios lo presenta de la siguiente manera:

\section{Sujeto y objeto:}

El sujeto es la persona, animal personificado o cosa con intención de conseguir lo que se ha propuesto. El objeto es la aspiración del sujeto, lo que suscita interés; puede ser la riqueza, la sabiduría, el amor, la felicidad, el aumento de sueldo.

\section{Destinador y destinatario:}

El destinador o dador suele ser una abstracción: la sociedad, el destino, el tiempo, etc., aunque puede encarnar a una persona. El destinatario suele coincidir con el sujeto, desea siempre algo o a alguien.

\section{Adyuvante y oponente:}

Inciden en la relación surgida entre el sujeto y el objeto, también determinan las aventuras y los obstáculos que ha de recuperar el sujeto. (Ayuso de Vicente, García \& Solano, 1990, p. 11).

Con estos elementos podemos realizar el análisis narratológico de la leyenda que nos ocupa.

\section{La bella de Puente Grande}

Hace muchos años vivía en Puente Grande un hombre muy católico llamado Felipe; era un campesino que atravesaba todos los días el puente para llegar a sus tierras. Un día, mientras marchaba de madrugada a trabajar, atravesó el puente y escuchó que alguien le llamaba por su nombre desde el río. La voz pertenecía a una mujer muy hermosa, muy blanca, que se bañaba completamente desnuda. Ella lo invitó a bañarse con él. Felipe, como todo hombre creyente y temeroso de Dios, desconfió de aquella mujer pecadora 
que de seguro era el diablo. Se persignó y encaró a la diabólica mujer para decirle que él era un hombre creyente y no caería en sus trampas infernales. Se dio la vuelta y se marchó; cuando volteó para asegurarse de que la mujer no lo siguiera, ella había desaparecido.

Como puede apreciarse, el relato es muy sencilo. El personaje no sufre transformaciones. Tiene el objeto y nunca lo pierde. (Para el modelo actancial, existen dos posiblidades: el sujeto "tiene" o "no tiene" el objeto). No hay, tampoco, una sucesión de estados que hagan evolucionar al protagonista, pues éste no experimenta transformaciones. Un estado se enuncia con los verbos ser, estar o tener. Sabemos que Felipe es hombre, que es un campesino y que es muy católico. Sabemos, también, que tiene trabajo y que está en camino para realizarlo.

¿Qué cambios se enuncia por medio de verbos de acción? Pocos: Felipe atraviesa el puente, escucha una voz que lo llama por su nombre, ve una hermosa mujer desnuda, desconfía de ella y piensa que es el diablo, se persigna, la encara, da la vuelta, se marcha, y vuelve a mirar para ver si lo sigue. Como puede verse, no hay un cambio de estado. El oponente no realiza un cambio en el sujeto, pues éste siempre posee al objeto.

\section{El esquema actancial puede ayudarnos a clarificar esta explicación:}

\begin{tabular}{|c|c|c|}
\hline DESTINATARIO & SUJETO & DFSTINADOR \\
\hline $\begin{array}{l}\text { Sistema de creencias } \\
\text { hegemónicas } \\
\text { Iglesia católica }\end{array}$ & Felipe & $\begin{array}{l}\text { Los hombres de bien } \\
\text { Comunidad católica }\end{array}$ \\
\hline ADYUVANTE & OBJETO & OPONENTE \\
\hline $\begin{array}{l}\text { Su fortaleza y la invocación a } \\
\text { la Santísima Trinidad (al }\end{array}$ & $\begin{array}{llr}\text { Permanecer } & \text { fiel a } & \text { sus } \\
\text { creencias y } & \text { vencer } & \text { las }\end{array}$ & $\begin{array}{l}\text { La mujer como tentación } \\
\text { diabólica encarnada }\end{array}$ \\
\hline
\end{tabular}




\begin{tabular}{|l|l|}
\hline persignarse) & tentaciones \\
\hline
\end{tabular}

Fuente: Elaboración propia

El objeto de Felipe es vencer la tentación y seguir siendo un hombre de bien. No se presta al engaño ni siente la mordedura del deseo carnal. Su oponente, pues, no logra ni siquiera inmutarlo. La invocación a la Santísima Trinidad es suficiente para ahuyentar al demonio (la mujer) que en este caso es inocuo. El destinatario, el sistema de creencias hegemónicas representado, en este caso, por la iglesia católica, dirige un mensaje muy claro, sin apenas matices, a los hombres de bien: no caigan en la tentación. Líbrense de ella de inmediato. En esta leyenda moral, el sujeto comprende lo que debe hacer, y además quiere, puede y sabe hacerlo.

\section{Análisis simbólico mítico}

Según Arnold Van Gennep (1982) debe entenderse por leyenda la narración localizada en espacios indicados con precision (en nuestro caso, Puente Grande), en un tiempo reconocible y cuyos actos parecen tener un fundamento histórico, pero que son objetos de fe:

[...] las leyendas son relatos simbólicos que dan cuenta de contenidos míticos. En tanto que plenas de símbolos, las leyendas son textos verbales que se iconizan para crear y recrear el imaginario común. La leyenda se construye a partir de la material prima de un mito: un referente histórico aparece transformado por la imaginación popular, se trata de una narracción ficcional transmitida de generación en generación. La acción aparece localizada en el espacio o en el tiempo, al contrario de los cuentos maravillosos, que se sitúan en un tiempo y espacio indeterminado. (García, 2007, p. 48).

Si la leyenda se construye a partir de la materia prima de un mito, ¿cuál, cualés están presentes en La bella de Puente Grande? En primer lugar, tenemos el agua como un elemento persistente en el discurso popular. Existen, y cualquier lector puede recordar más de un caso, numerosas tradiciones míticas en donde mujeres (y hombres, claro) surgen de un lago, un río o una fuente. También hay 
descensos al mundo subterráneo o a realidades alternas a través del agua, que sirve como un elemento de transición entre el mundo real y el fantástico.

Habrá que recordar a las sirenas, que son demonios con figura de mujer, tentaciones encarnadas que atraían a los marineros para destruirlos. En la Antigua Grecia se las describía como mujeres-ave de presa, es verdad, pero elaboraciones posteriores las convirtieron en un pecessirena, y esta es la imagen que se ha quedado en el imaginario popular.

Más cercana a las características de la mujer demonio de Puente Grande es la rusalka de la mitlogía eslava, pues se trata de una sirena, digamos, de agua dulce. La rusalka es también un demonio con forma de mujer que atrae a los hombres con canciones y bailes para conducirlos luego al fondo del río. Se dice que, incluso, oír su risa puede provocar la muerte.

La tradición cristiana, por su parte, ha sido pródiga en relatos de mujeres tentadoras que provocan la perdición de los hombres de bien. Las páginas de Historias bizantinas de locura y santidad, de Juan Mosco y Leoncio de Neápolis (2005), por poner un ejemplo, están llenas de relatos de hombres de bien (anacoretas, ermitaños que viven en cuevas y vagan por las montañas) que han sido atacados por el demononio de la fornicación, por la lujuria. El imaginario cristiano popular admite seres antropomorfos con cuernos y rabo. Diablos feos, color negro o rojo, cuyas deformaciones físicas son reflejo de su monstruosidad ontológica. Pero también "sabe" que las mujeres hermosas están casadas con el diablo. Y que el diablo se aprovecha de que la carne es débil para perdernos.

Al cristianismo le debemos, pues, la demonización del deseo carnal. Es verdad que los griegos aconsejaban mantener la compostura, y que consideraban el sexo y el desperdicio de la simiente como una pérdida de energía para el hombre superior, pero debemos a los cristianos la advertencia de que el deseo o el placer por sí mismos son causa de ruina. Los grandes hombres que renuncian a arder, que viven en castidad y retiro, se ven agobiados por tentaciones pecaminosas. San Jerónimo nos cuenta su experiencia con estas palabras: 
Allí estaba yo, pues, condenado por mí mismo a aquella prisión por miedo al infierno, con sólo los escorpiones y las bestias salvajes por compañeros. Y sin embargo a menudo me veía rodeado de bailarinas. Tenía el rostro pálido por el ayuno, y mi mente ardía de deseo dentro de un cuerpo frío como el hielo. Aunque mi carne, adelantándoe a su inquilino, estuviera ya prácticamente muerta, los fuegos de las pasiones seguían ardiendo en mi interior.

Y así, desamparado de toda ayuda, me encontraba a los pies de Jesús. Los bañaba con mis lágrimas, los limpiaba con mi pelo. Cuando mi carne se rebelaba, la sometía con semanas de ayuno. (Blackburn, 2005, pp. 72-73).

Un santo sufre por verse rodeado de tentadoras bailarinas, que podemos imaginar desnudas. El cristianismo no da tregua: también peca quien imagina, se ilusiona o sueña, pues cualquiera que mire a una mujer con lujuria ha cometido adulterio con ella en su corazón, dice el Señor. El Ecliesiastés va por el mismo tono "El pecado comenzó por la mujer y por ella todos moriremos".

Para el cristianismo, disfrutar del sexo es pecado mortal. El placer carnal debe ser desechado por quien tenga algún respeto por sí mismo. El buen cristiano debe procurar no hacer nada que distraiga su pureza, así que está obligado a apartarse por completo de toda tentación. Las mujeres hermosas deleitan deshonestamente y provocan la lujuria. El hombre recto no debe hacerse ilusiones creyendo que puede moderar la tentación fijando un límite para no sobrepasarse. Quien piense eso se engaña a sí mismo, pues la tentación impura es como un incendio: si se le deja tomar fuerza llega a ser imposible dominarlo. La mirada de una mujer se prende al corazón y se mete en todos los rincones del alma, aniquilando espiritualmente al hombre de buena voluntad. No resulta extraño que los hombres de bien, como Felipe, se feliciten por haber resistido la tentación del abismo.

Así pues, en resumen: la asociación de la mujer con la lujuria, el mal, la condenación y el infierno está firmemente asentada en la tradición cristiana católica. Y hay libros, sermones, relatos, novelas, cuentos, poemas y leyendas que nos advierten de ello. 


\section{Conclusiones}

Felipe puede seguir con su vida en paz, pues la posible tentación, el diablo con forma de mujer, se esfuma. El destinador del relato, es decir, el conjunto de convenciones y creencias vigentes de la iglesia católica, ha logrado su cometido: fijar un camino que la comunidad debe seguir. Si las leyendas son patrimonio inmaterial, sustrato de memoria colectiva, es porque son portadoras de un pensamiento grupal, de una constelación de valores y juicios, es decir, de una visión del mundo. $L a$ bella de Puente Grande nos da una imagen de nosotros mismos, nos permite reconocernos y construir nuestra realidad. ¿Qué realidad nos muestra? Una en la que los hombres son capaces de fuerza de voluntad y de ejecución y las mujeres inducen al mal. Por otra parte, el hecho de que la tentadora sea muy blanca y, por lo tanto, hermosa, refuerza un canon malinchista de belleza que ve "en la blancura la mitad de la hermosura". Pero también revela que debemos ver con desconfianza a estas mujeres que se distinguen del resto por su físico y por sus actitudes, pues son causa de pena y perdición. Así pues, la leyenda propone un prototipo negativo de la mujer, asociándola con la tentación del placer carnal y el deseo impuro que conduce a la perdición. Un estereotipo muy antiguo, como podemos apreciar por las resonancias de las mitologías griega y eslava. Una herencia de siglos.

Al estilo de Facundia Ratis, de Egberto de Lieja, publicado en el siglo XI, un libro de enseñanzas cristianas, didáctico y catequizante, las leyendas de advertencia moral rigen las creencias, las jerarquías y las reglas que debe seguir un pueblo. Señala cómo se debe vivir. Cabe destacar que esta imagen de la mujer no es un caso aislado en el corpus de leyendas que son patrimonio cultural de Tonalá; otras leyendas, como las de Don Guitarrón Cascado y La ancianita del Cerro de la Reina también muestran que el mundo es de los hombres, que en ellos está la fuerza de ejecución. Las mujeres, o intervienen en el papel de tentadoras, porque inducen al mal, o son muy buenas pero incapaces de acción, así que deben ser salvadas. Queda pendiente, para futuras 
investigaciones, el análisis literario simbólico mítico de estas leyendas y otros relatos populares de

Tonalá, que nos revelará la visión del mundo que transmiten en conjunto.

\section{Referencias:}

Aurelio, G. (2004). Leyendas de Tonalá. Tonalá: Robles impresiones.

Ayuso, M., García, C. \& Solano, S. (1990). Diccionario de términos literarios. Madrid: Akal.

Blackburn, S. (2005). Lujuria. Madrid: Paidós.

García, L. (2007). Etnoliteratura. Principios teóricos para el análisis simbólico del imaginario simbólico-mítico. Colima: Universidad de Colima, Colima.

Marcelli, J. Pelayo, I. Hermosillo, A. (2017). Escenarios intangibles: La cultura literaria, sonora y artística de Tonalá. (En proceso de dictaminación).

Mosco, J. \& Néapolis de, L. (2005). Historias bizantinas de locura y santidad Madrid: Siruela.

Pérez, H. (2001). "Tradición y oralidad en el refranero mexicano" en La tradición hoy en día. Primer foro interdisciplinario de oralidad, tradición y culturas populares y urbanas. Memorias. México, UIA, 15-70.

Van Gennep, A. (1982). La formación de las leyendas. Barcelona: Alta Fulla. 